2. Aicher D, Langer F, Kissinger A, Lausberg H, Fries R, Schäfers HJ. Valve-sparing aortic root replacement in bicuspid aortic valves: a reasonable option? J Thorac Cardiovasc Surg. 2004;128:662-8

3. Schäfers HJ, Langer F, Aicher D, Graeter T, Wendler O. Remodeling of the aortic root and reconstruction of the bicuspid aortic valve. Ann Thorac Surg. 2000;70: $542-6$
4. De Paulis R, De Matteis GM, Nardi P, Scaffa R, Bassano C, Chiariello C. Analysis of valve motion after the reimplantation type of valve-sparing procedure (David I) with a new aortic root conduit. Ann Thorac Surg. 2002;74:53-7.

5. Grande KJ, Cochran RP, Reinhall PG, Kunzelman KS. Mechanics of aortic valve incompetence: finite element modeling of aortic root dilatation. Ann Thorac Surg. 2000;69:1851-7.

\title{
Maintaining situational awareness in a cardiac intensive care unit
}

\author{
Daniel Engelman, MD, ${ }^{\mathrm{a}}$ Thomas L. Higgins, MD, ${ }^{\mathrm{b}}$ Rakesh Talati, MD, ${ }^{\mathrm{a}}$ and Jason Grimsman, MD, ${ }^{\mathrm{a}}$ \\ Springfield, Mass
}

We have developed a computerized system to assimilate pertinent real-time and historical patient data and present it in a user-oriented, clinically relevant form in our cardiac intensive care unit (ICU). This display is projected continuously above each patient's bed on a large liquid crystal display screen and updated in real time. This allows a provider team to simultaneously speak with a patient while reviewing all pertinent laboratory values, vital signs, ventilator settings, intake and output, hemodynamics, and vasoactive drug dosages, with trends shown in a graphic format. We believe this facilitates efficient, high-quality care and maintains optimal situational awareness.

Caring for patients in an ICU requires the assimilation of large amounts of continuously fluctuating data. The relationship between the various laboratory results, vital signs, medication dosages, and fluid shifts across time is an essential component to maintaining situational awareness of a patient's condition. This requires a practitioner to look at a huge variety of data, determine what is relevant, synthesize the data, and act on the analysis. In a modern ICU, this requires the detection and interpretation of cues from multiple dynamically changing data streams and the rapid adaptation to an evolving clinical situation. The need for a situational awareness tool in an ICU setting has been previously documented. ${ }^{1}$

Historically, situational awareness in many ICUs was maintained by means of an extensive handwritten flow sheet with hundreds of pieces of data manually added horizontally along a time axis. The clinician would assimilate how

\footnotetext{
From the Divisions of Cardiac Surgery ${ }^{\mathrm{a}}$ and Critical Care, ${ }^{\mathrm{b}}$ Baystate Medical Center, Springfield, Mass.

Disclosures: Daniel Engelman reports consulting and lecture fees from Cadence Pharmaceutical, and consulting fees from St. Jude Medical. All other authors have nothing to disclose with regard to commercial support.

Received for publication March 10, 2013; revisions received Oct 21, 2013; accepted for publication Oct 25, 2013; available ahead of print Dec 2, 2013.

Address for reprints: Daniel Engelman, MD, Division of Cardiac Surgery, Baystate Medical Center, 759 Chestnut St, Suite 4628, Springfield, MA 01107 (E-mail: daniel.engelman@baystatehealth.org).

J Thorac Cardiovasc Surg 2014;147:1105-6

$0022-5223 / \$ 36.00$

Copyright (c) 2014 by The American Association for Thoracic Surgery

http://dx.doi.org/10.1016/j.jtcvs.2013.10.044
}

changes in a given variable affected others across time. As electronic medical records have become standard, however, these handwritten flow sheets are being eliminated. This has necessitated ICUs to shift to computerized data gathering, which is often limited by screen size and by various menu-driven data sets of variable hospital information systems. Providers are at risk of losing situational awareness because the data are segregated onto different screens. In addition, it is difficult to assimilate all the data simultaneously into an overall clinical snapshot. Finally, the information requires logging into a nursing station computer, which may be out of the reach of the bedside clinician. There is little ability to talk to a patient or the rounding clinical team while simultaneously reviewing data from a password-protected information system.

As critical care technology has become more sophisticated, each device has fed its own monitoring system. This has resulted in a technology-centered design contributing to information overload. As an example, each of our postoperative cardiac surgical patients has separate monitors displaying vital signs, cardiac index, ventilator and intravenous pump settings, and laboratory results. There are also displays of the outputs from the drainage containers for chest tubes, urimeters, and nasogastric tubes. Finally, chest radiographs and continuous electrocardiographic monitoring are recorded. For a clinician to assimilate all this information accurately, this requires viewing multiple monitors and filtering large amounts of often superfluous data. A better system would be one that is user centered. ${ }^{2}$ This should be a presentation of relevant data that is goal directed, with only clinically relevant data included and presented to the clinician in a comprehensible and easily recognized form.

Our new system graphically displays large amounts of real-time patient data from multiple sources. The information is displayed over the patient's bed, allowing the clinical team to peruse key data while conversing in the patient's room during rounds (Figure 1). This placement also results in a more "patient-centric" and open discussion with family members. The graphic user interface is specifically designed to provide the maximal information for clinicians 


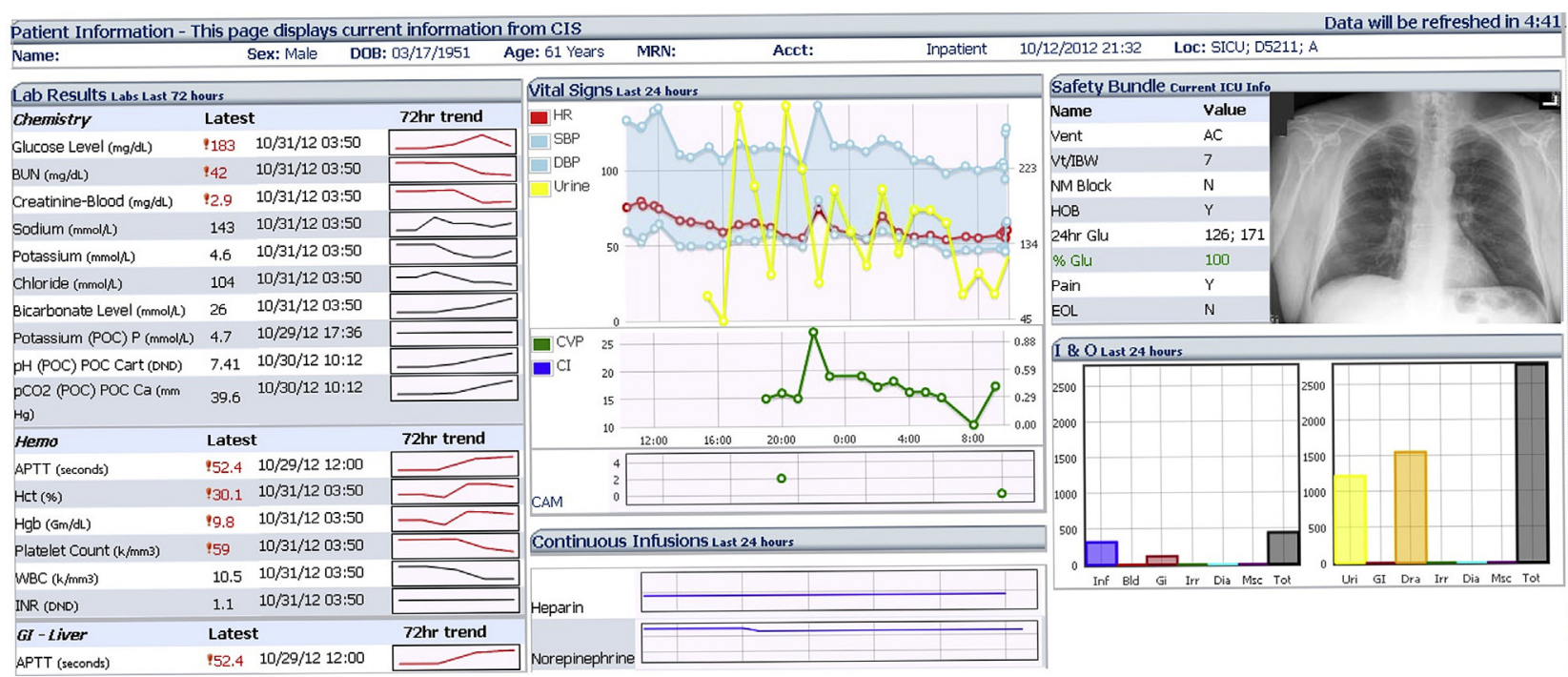

FIGURE 1. A real-time, bedside graphic display of patient data from multiple sources allows the clinical team to peruse key data while conversing in the patient's room during rounds.

to note the most recent clinical information and historical trends. All data are sequenced, allowing one to determine rapidly how variables affected one another across time. These are displayed with key warning colors and ranges to facilitate the review of both historical and real-time data. It has been previously demonstrated that the assimilation of data is faster when presented in a graphic format rather than in a data table. ${ }^{3}$

To avoid redundancy, all the data graphically displayed are pulled directly from the electronic medical record. This is accomplished by extracting the necessary data and placing them in a database to be used for the graphic display. These data are refreshed every 5 minutes to keep the situational awareness as close to real time as possible. This graphic format is displayed on a 42-inch liquid crystal display screen mounted at the head of the bed. It is driven by a desktop computer that uses a web browser running in full-screen mode to display the data as a patient-specific Web page built on HTML, XML, and JavaScript from the data pulled from the electronic medical record.

The system is continuously being modified to incorporate proprietary warning notifications to allow clinicians to act proactively rather than reactively to a patient's deteriorating condition. The triggers for these warnings include both prespecified percentage changes in laboratory values with time and new abnormal laboratory results. Examples of such warnings include a $50 \%$ drop from baseline in the platelet count after a heparin exposure and a new positive microbiology result. Finally, customizable measures, such as 24-hour postoperative glucose levels and time from intubation, are highlighted to focus attention on specific inpatient quality measures. These measures are based on
Society of Critical Care Medicine and Society of Thoracic Surgeons recommendations.

In the 6 months since the introduction of this system, we have noted significant decreases in postoperative renal failure, blood product use, glucose control problems, and neurologic complications, as defined by the Society of Thoracic Surgeons database. Although the monitoring system may not be independently responsible for these improvements, we theorize that an earlier appreciation of relevant data may at least be contributory. As an example, nephrotoxic drugs can be discontinued at the first sign of renal dysfunction. In a blinded survey, $55 \%$ of our nursing and midlevel staff stated the system had provided early warning information that otherwise would have been missed with the standard electronic medical record. In surveys, both practitioners and patient families believed that the system improved the overall process of care.

The situational awareness display is being continuously modified in collaboration between the medical informatics team and the bedside practitioners through a prototype screen in a designated patient room. We believe this system will result in a reduction in medical errors as providers are given information allowing them better to understand clinically relevant material and predict problems before they happen.

\section{References}

1. Koch SH, Weir C, Haar M, Staggers N, Agutter J, Görges M, et al. Intensive care unit nurses' information needs and recommendations for integrated displays to improve nurses' situation awareness. J Am Med Inform Assoc. 2012;19:583-90.

2. Endsley MR. Designing for situation awareness: an approach to user-centered design. 2nd ed. Boca Raton (FL): CRC Press; 2011.

3. Bauer DT, Guerlain S, Brown PJ. The design and evaluation of a graphical display for laboratory data. J Am Med Inform Assoc. 2010;17:416-24. 